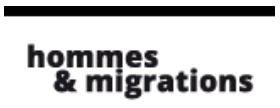

\section{Hommes \& migrations}

Revue française de référence sur les dynamiques

migratoires

1303 | 2013

Diasporas marocaines

\title{
Diasporas et développement
}

\section{Jean-Baptiste Meyer}

\section{(2) OpenEdition \\ Journals}

\section{Édition électronique}

URL : http://journals.openedition.org/hommesmigrations/2571

DOI : 10.4000/hommesmigrations. 2571

ISSN : 2262-3353

\section{Éditeur}

Musée national de l'histoire de l'immigration

\section{Édition imprimée}

Date de publication : 1 juillet 2013

Pagination : $134-137$

ISBN : 978-2-919040-23-0

ISSN : $1142-852 X$

\section{Référence électronique}

Jean-Baptiste Meyer, « Diasporas et développement », Hommes \& migrations [En ligne], 1303 | 2013, mis en ligne le 31 décembre 2015, consulté le 02 mai 2019. URL : http://journals.openedition.org/ hommesmigrations/2571; DOI : 10.4000/hommesmigrations.2571 


\section{DIASPORAS ET DÉVELOPPEMENT}

par JEAN-BAPTISTE MEYER, sociologue, directeur de recherches à l'IRD

Comment cultiver les liens et les talents de la diaspora pour des activités créatrices? De quelle façon réaliser leur potentiel pour le développement des pays qui les concernent? La question est en haut de l'agenda des politiques de coopération tant en matière migratoire que de développement. De nombreuses réunions multilatérales se tiennent en cette fin d'année 2013 pour traiter de la question. Le 20 juin dernier, la première rencontre interministérielle sur les diasporas, réunie par l'Organisation internationale pour les migrations (OIM), a vu le jour à Genève. Début octobre, à New York, se tiendra le dialogue de haut niveau des Nations unies sur les migrations et le développement.

L’année 2014 sera l'occasion d'intenses réflexions et de débats nombreux sur l'agenda du développement après 2015 et l'achèvement des objectifs du millénaire. La Suède mobilise le Forum mondial sur les migrations et le développement dans cette perspective, avec l'inclusion de la mobilité dans les questions fondamentales de la coopération planétaire. À chaque fois, la diaspora apparaît comme un élément positif, un potentiel de transfert, de fertilisation croisée, entre des aires culturelles, sociales et économiques disparates. Cette vision favorable de la diaspora est aujourd'hui largement partagée par les États du monde, qui y voient un moyen de déployer leurs ressources humaines au-delà des frontières du territoire national. C'est un phénomène historique : celui de la mondialisation de l'État-nation.
Des politiques de la diaspora ont vu le jour, soutenues par des études; des manuels ou des guides, généralement produits par les organisations internationales, se sont multipliés (voir bibliographie ciaprès). Ils conseillent, orientent ou indiquent des dispositions à prendre pour tirer parti des diasporas dans des optiques de développement.

\section{Développer la connaissance des réseaux diasporiques}

L'idée générale des politiques de diaspora consiste à mobiliser les expatriés pour transférer et traduire des ressources accumulées d'un côté et en faire bénéficier l'autre, plus dépourvu. Aujourd'hui, les liens sont avérés et prometteurs mais on ignore encore beaucoup des conditions propices à des réalisations effectives. L'abondante littérature produite récemment pour prescrire les bonnes pratiques des politiques de la diaspora tente de pallier ce déficit d'information et d'établir empiriquement les voies de la réussite. Mais elle se heurte à une contrainte paradoxale : les réseaux de la diaspora sont, à l'évidence, transnationaux. Leur constitution et leur appréhension transcendent le cadre, les catégories et les instruments des politiques publiques traditionnelles relevant de l'État-nation. Il est par conséquent nécessaire de rechercher des voies nouvelles, en marge de ces approches traditionnelles, 
plète et détaillée possible, sur les expatriés et leurs réseaux. Où sont-ils ? Que font-ils ? Comment se réunissent-ils ? Que projettent-ils ? On sait ainsi aujourd'hui, par exemple, que les ressources humaines argentines sont plus présentes en Europe quailleurs et celles de la Colombie plus denses dans le business aux États-Unis que dans l'innovation scientifique et technique ; les actuelles circulations et les retours croissants des Uruguayens se dirigent toujours beaucoup vers la capitale, Montevideo. La gestion de ces mouvements complexes s'améliore avec la connaissance accrue rendue disponible par cet observatoire (www.observatoriodiasporas.org). Elle permet de mieux savoir où porter l'attention, quel effort peut être promu et quelle priorité doit être défendue. Au-delà de cette information précise mais descriptive, des bases de données concernant des milliers de personnes ont été dressées.

Coopérative de femmes pour la fabrication de l'huile d'argan, Taroudant, Maroc, mai 2001 @ Charles Carrié

pour explorer, expliquer et gérer les liens diasporiques avec des visées développementales. Dans ce but a été conçu et mené le projet Cidesal (Création d'incubateurs de diasporas du savoir pour l'Amérique latine). Il part du constat que les liens diasporiques ne vont pas de soi, même si une dynamique spontanée des expatriés se fait jour et que les entités publiques la voient d'un œil favorable. Établi sur des conclusions d'études empiriques antérieures de l'IRD ainsi que sur celles de l'Unesco et d'autres organisations, il propose des dispositifs d'accompagnement et de renforcement de ces liens. Cidesal a ainsi expérimenté, pour l'Amérique latine et ses expatriés hautement qualifiés, des modalités d'incubation des réseaux diasporas, visant à les développer et à en faciliter les actions productives.

La première chose à faire, tous les experts s'accordent sur ce point, c'est de bien connaître la diaspora. C'est par là qu'a commencé le projet Cidesal : produire une information originale, la plus com-
Elles donnent un possible premier accès à ces ressources humaines expatriées identifiées. Les personnes qui constituent ces diasporas sont ainsi contactables et potentiellement mobilisables, selon les données et les coordonnées fournies.

Mais un constat s'impose aussi : la partie visible et naturellement accessible de la diaspora est extrêmement minoritaire. On pensait auparavant que les réseaux consulaires, institutionnels et associatifs permettaient d'identifier et de localiser une grande partie de ces populations. Or un sondage très précis révèle que seulement 10 à $20 \%$ des expatriés susceptibles de se connecter entre eux et avec le pays relèvent de ces réseaux formels. Le défi consiste donc à trouver tous les autres, audelà de ces canaux traditionnels, d'en percevoir le profil de compétences, de pouvoir les contacter de façon circonstanciée, pour concevoir éventuellement des actions concertées. Il faut donc aller au-delà des recensements systématiques mais toujours limités et des résultats stockés dans des bases de données vite obsolètes du fait de la volatilité des acteurs diasporiques. Le but est de se brancher sur 
le Web et de sonder par des techniques de datamining les champs où peuvent se détecter des traces de mobilité en rapport avec des pays ou régions à la recherche de ces ressortissants éloignés.

\section{À l'heure des diasporas 2.0}

Pour relever ce défi, une équipe d'informaticiens du projet Cidesal a créé un système de logiciels intégrés, capable de rechercher, un par un, les profils diasporiques spécifiques. Cet outil s'appelle Unoporuno (https://github.com/unoporuno/ unoporuno). À partir d'un corpus de noms et de quelques champs renseignés, il fouille le Web à la recherche d'indices permettant de retracer l'origine et l'itinéraire des individus, d'explorer leur composante professionnelle, sociale et cognitive et de relever leurs coordonnées. Le logiciel est en accès libre et à disposition de tous les acteurs, particuliers ou organisations,

Des plateformes électroniques ont été construites, offrant différentes fonctions de communication spécialisée : forums, dépôt de CV, vidéos, conférences présencielles ou virtuelles, échange de documents. le spectre d'utilisation aux individus comme aux institutions. Mais c'est l'orientation vers une mobilisation circonstanciée qui confère à Unoporuno son utilité. Les personnes identifiées, localisées et caractérisées peuvent être démarchées en toute connaissance de cause. Elles sont approchées, non pas de loin et pour des motifs généraux ou aléatoires, mais selon leurs compétences, leur insertion socioprofessionnelle, leurs relations intellectuelles ou diplomatiques. Cette opportunité d'un appariement très fin des acteurs pour réaliser ensemble des opérations sophistiquées ouvre des perspectives inédites. Cependant, une fois ces partenariats identifiés, comment leur donner vie ? Où peuvent-ils trouver l'environnement de premiers contacts à distance? Un espace d'interaction où déployer ces relations potentielles et commencer à les pratiquer a été créé par le projet Cidesal. Des plateformes électroniques ont été construites, offrant différentes fonctions de communication spécialisée : forums, dépôt de $C V$, vidéos, conférences présencielles ou virtuelles, échange de documents. La constitution de groupes thématiques a été encouragée sous l'égide de responsables autodésignés, ou pressentis et contactés par les "community managers" de ces plateformes, la red C (http://laredc.com/) et red uruguay encuentro (http://reduruguayencuentro.com/).

Les réseaux sociaux qui émergent par ces mises en contact vont au-delà de ceux qui existent par ailleurs. Une enquête réalisée auprès des milliers de participants à ces plateformes - colombienne et uruguayenne - indique qu'une majorité d'entre eux vient pour créer du contact et à l'occasion, à travers elles, tisser de nouveaux liens. Cela est très différent des réseaux scientifiques traditionnels ou institutionnels, émanant par exemple de programmes gouvernementaux comme Raices, en Argentine, où les structures digitales viennent en fait seulement alimenter des relations sociales préexistantes. On se demande souvent, en effet, si de nouvelles connexions sont utiles aux personnes déjà largement intégrées à des réseaux internationaux comme le sont les professionnels et tout particulièrement les chercheurs et ingénieurs. L'expérience menée par Cidesal prouve l'intérêt que revêtent ces espaces aménagés. Ils sont bien l'occasion de matérialiser des propensions à l'identification vers des origines nationales, qui resteraient autrement en latence. Mais on discerne aussi l'extrême labilité de ces réseaux. Les relations tissées redeviennent vite invisibles et se développent volontiers en dehors des plateformes une fois le contact fait. 
Il y a là tout un spectre de relations inédites qui apparaissent à travers ces nouvelles conditions de communication. Ce sont des liens décentralisés, produits par l'interaction directe ménagée par les réseaux informatiques. Il n'y a pas de gestion dirigiste de ces relations mais seulement une stratégie pour en favoriser l'éclosion, le développement et l'application. Le rôle de l'État, dans le pays d'origine mais aussi dans celui d'accueil, consiste à faciliter la mise en place de ces infrastructures informationnelles et à fournir des programmes où les actions possibles entre diasporas et communautés locales soient favorisées, au moins sur les secteurs et dans les domaines reconnus d'utilité nationale. Cela signifie que les ressources humaines de la diaspora doivent être conçues et intégrées depuis le début dans les stratégies de développement.

La politique publique correspondant à cette gouvernance consiste à faciliter l'implication de la diaspora sans lui assigner un rôle de façon directive. C'est en l'incluant (juridiquement, politiquement) comme acteur que l'État pourra compter sur ses services. Mais son association au cas par cas sur des projets nécessitera pour se réaliser le maillage social intelligent que fournissent des dispositifs informatiques tels que ceux proposés par Cidesal. Ce ne sont pas des instruments magiques mais des modalités nouvelles de mise en relation. Ils requièrent un apprentissage et une pratique assidues pour être efficaces.

Pour cette raison, la métaphore du tango correspond à ce mode de gouvernance : une technique sophistiquée, une coordination collective par les instruments et la pratique, sans chef d'orchestre mais avec beaucoup de connaissance. Dans une telle configuration, on ne gouverne plus par l'administration, dont une population avec des référents territoriaux constituerait l'objet d'exercice, mais à travers un pilotage du social, où les acteurs sont également sujets, avec le même accès que leurs administrateurs aux dispositifs qui régissent leurs relations.

\section{Références Bibliographiques}

- Dovelyn Agunias, Kathleen Newlands, Developing a Road Map for Engaging Diasporas in Development, a Handbook for Policy Makers and Practitioners in Home and Host Countries, International Organization for Migrations, Washington DC, Migration Policy Institute, 2012.

- Kingsley Aikins, Nicola White, Global Diaspora, Strategies Tool Kit. Harnessing the Power of Global Diasporas, Dublin, Diaspora Matters, 2012.

- Institut de recherche pour le développement (IRD), Scientific Diasporas/Diasporas scientifiques, Paris, IRD, 2003.

- Yevgeny Kuznetsov, Diaspora Networks and the International Migration of Skills. How countries can Draw on their Talents Abroad?, Washington DC, World Bank Institute, 2006.

- Organisation de coopération et de développement économiques.

- Organisation de coopération et de développement économiques (OCDE), Harnessing the Skills of Migrants and Diasporas to Foster Development. Policy Options, Paris, Direction générale de la mondialisation, du développement et des partenariats, ministère des Affaires étrangères, 2012.

- Organisation de coopération et de développement économiques (OCDE), Agence française de développement (AFD), Connecting with Emigrants. A Global Profile of Diasporas, Paris, 2012.

- Gabriela Tejada, Jean-Claude Bolay, An Action Oriented Tool Kit to Assess Good Practices of Skilled Migrants and Scientific Diasporas, Cooperation EPFL, Lausanne, 2010.

- United Nations Conference on Trade and Development (UNCTAD), Harnessing Remittances and Diaspora Knowledge to Build Productive Capacities; the least developed countries report, Genève, 2012. 